\title{
The bound dynamics of the same polarity 180-degree domain walls in biaxial ferromagnets
}

\author{
V.N. Nazarov ${ }^{1, \dagger}$, E.G. Ekomasov ${ }^{2}$ \\ †NazarovVN@gmail.com \\ ${ }^{1}$ Institute of Molecule and Crystal Physics Ufa Research Center of RAS, Prospekt Oktyabrya 151, Ufa, Russia, 450075 \\ ${ }^{2}$ Bashkir State University, 32, Validy Str., 450076, Ufa, Russia
}

The bound dynamics of two $180^{\circ}$ domain walls with the same polarity and opposite topological charges is investigated theoretically in a biaxial ferromagnet with a large quality factor. The domain walls moving towards each other under the influence of an external magnetic field are considered taking dissipation into account. The case of a large size ferromagnet under small external magnetic field and attenuation has been studied. An inhomogeneous exchange interaction, uniaxial and rhombic anisotropy are taken into account in the magnetic energy density. A soliton model with one spatial coordinate, in which the interacting domain walls are regarded as a two-soliton solution, is used. A system of ordinary differential equations describing the evolution of the soliton solution parameters under the influence of the magnetic field and attenuation is obtained. The numerical study of the differential equations shows a strong dependence of the interaction of walls in the magnetic field on the attenuation. It was found that during the interaction of two 180-degree walls of the same polarity at a low attenuation and specific direction of the external magnetic field there occurs an annihilation of the walls, accompanied by multiple scattering process and the walls polarity change. With the increase of the attenuation parameter the annihilation of soliton-antisoliton pair happens as a result of a single scattering process. In the case of the field of the opposite direction, same polarity 180-degree domain walls, while moving, come at the minimum distance and then repel each other and move away from each other.

Keywords: magnetic solitons dynamics, biaxial ferromagnet.

\section{Связанная динамика 180-градусных доменных стенок одинаковой полярности в двухосных ферромагнетиках}

\author{
Назаров В.Н. ${ }^{1, \dagger}$, Екомасов Е.Г. ${ }^{2}$ \\ ${ }^{1}$ Институт физики молекул и кристаллов УНЦ РАН, пр. Октября, 151, 450075, Уфа, Россия \\ ${ }^{2}$ Башкирский государственный университет, ул. 3. Валиди, 32, 450074, Уфа, Россия
}

Теоретически исследуется связанная динамика двух 180-градусных доменных стенок одинаковой полярности с противоположными топологическими зарядами в двухосном ферромагнетике с большим фактором качества. Рассматриваются движущиеся навстречу друг другу доменные стенки под действием внешнего магнитного поля с учетом диссипации. Рассмотрен случай ферромагнетика больших размеров, малых внешних магнитных полей и затуханий. В плотности энергии магнетика учтено неоднородное обменное взаимодействие, одноосная и ромбическая анизотропии. Используется одномерная по координате солитонная модель, в которой взаимодействующие доменные стенки рассматриваются как двухсолитонное решение. Получена система обыкновенных дифференциальных уравнений, описывающая эволюцию параметров солитонного решения под действием магнитного поля и затухания. Численное исследование дифференциальных уравнений показывает сильную зависимость процесса взаимодействия стенок в магнитном поле от затухания. Установлено, что при взаимодействии двух 180-градусных стенок одинаковой полярности при малом затухании и определенном направлении внешнего магнитного поля имеет место аннигиляция стенок, сопровождающаяся многократным процессом рассеяния и сменой полярности стенок. Максимальное расстояние между стенками, на которое они расходятся после взаимодействия, зависит от значения внешнего поля и параметра затухания. В результате взаимодействия кинка и антикинка фазовый сдвиг имеет хорошую зависимость от фактора качества материала. С увеличением параметра затухания аннигиляция солитон-антисолитонной пары происходит в результате одноактного процесса рассеяния. В случае противоположного направления поля при движении 180-градусные доменные стенки одинаковой полярности приближаются на минимальное расстояние, после чего происходит их отталкивание и последующее разбегание друг от друга.

Ключевые слова: динамика магнитных солитонов, двухосный ферромагнетик. 
Известно, что кроме односолитонного решения, описывающего стационарно движущуюся 180-градусную доменную стенку, уравнение Ландау-Лифшица допускает как двухсолитонные, так и многосолитонные решения [1 - 3]. Многосолитонные решения описывают уже взаимодействующие доменные стенки, которые играют главную роль в процессе неоднородного перемагничивания. Перемагничивание один из самых важных процессов связанный с ферромагнитными материалами и его исследования не прекращаются [см., например, 4-6]. Перемагничивание может сопровождаться образованием зародыша перемагничивания с последующим преобразованием его в домен обратной намагниченности [7], ограниченный двумя взаимодействующими доменными стенками. Далее этот процесс сопровождается взаимодействием движущихся навстречу друг другу доменных стенок. При этом взаимодействующие доменные стенки одинаковой и противоположной полярности ведут себя по разному. В случае доменных стенок одинаковой полярности имеет место их аннигиляция, а разной полярности - образование динамической 360-градусной доменной стенки. Взаимодействие доменных стенок исследовались в материалах с малым фактором качества [8] и в легкоплоскостном ферромагнетике [9]. Влияние внешнего магнитного поля и диссипации на двух- и многосолитонные решения исследовано еще не достаточно полно. Это связано с тем, что учет затухания и внешнего магнитного поля нарушает интегрируемость уравнения Ландау - Лифшица. Влияние диссипации на движение двухпараметрического солитона в легкоосном ферромагнетике без учета магнитодипольного взаимодействия в однородном магнитном поле изучалось в работе [10]. Однако такое приближение не позволяет описать особенности динамики взаимодействующих доменных стенок одинаковой и разной полярности в ферромагнетиках с конечной величиной фактора качества, а также в двухосных ферромагнетиках. Настоящая работа посвящена теоретическому исследованию взаимодействия 180-градусных стенок одинаковой полярности с противоположными топологическими зарядами в двухосном ферромагнетике с большим фактором качества во внешнем магнитном поле с учетом диссипации.

Рассмотрим ферромагнетик больших размеров, для плотности энергии которого запишем выражение [3]:

$$
w=A\left(\frac{\partial \mathbf{m}}{\partial y}\right)^{2}-K_{u} m_{z}^{2}+\left(K_{p}+2 \pi M_{S}^{2}\right) m_{y}^{2}-M_{S} \mathbf{H m},
$$

где $A-$ параметр неоднородного обменного взаимодействия; $\mathbf{m}=\mathbf{M} / M_{\mathrm{S}}$ - единичный вектор намагниченности $\mathbf{M}, M_{\mathrm{S}}=|\mathbf{M}|-$ намагниченность насыщения; $K_{\mathrm{u}}$ - константа одноосной анизотропии; $K_{\mathrm{p}}-$ константа ромбической анизотропии, $\mathbf{H}=(0,0,-H)$ - внешнее магнитное поле. При $K_{\mathrm{u}}>0, K_{\mathrm{p}}>0$ в основном состоянии намагниченность ориентируется параллельно оси $z$, т.е. $\mathbf{M} \| z$.

Уравнение Ландау-Лифшица-Гильберта с учетом (1) в угловых переменных $\mathrm{m}=(\sin \theta \cos \varphi, \sin \theta \sin \varphi, \cos \theta)$ можно представить в виде

$$
\theta_{\xi \xi}-\left(1+\varphi_{\xi}^{2}+\varepsilon \sin ^{2} \varphi\right) \sin \theta \cos \theta+h \sin \theta=\alpha \theta_{\tau}-\varphi_{\tau} \sin \theta,
$$

$\left(\varphi_{\xi} \sin ^{2} \theta\right)_{\xi}-\varepsilon \sin ^{2} \theta \sin \varphi \cos \varphi=\theta_{\tau} \sin \theta+\alpha \varphi_{\tau} \sin ^{2} \theta$.
Здесь $\xi=y / \delta_{0}, \delta_{0}=\sqrt{A / K_{u}}, \varepsilon=\left(2 \pi M_{S}^{2}+K_{p}\right) / K_{u}, \tau=\omega_{F M} t-$ безразмерное время, $\omega_{F M}=2 \gamma / M_{S}\left(K_{u}\left(K_{u}+K_{p}+2 \pi M_{S}^{2}\right)\right)^{1 / 2}-$ частота ферромагнитного резонанса, $h=M_{s} H / 2 K-$ безразмерное поле, $\gamma$ - гиромагнитное отношение, $\alpha-$ параметр затухания. Используем далее солитонную модель, в которой взаимодействующие доменные стенки рассматриваются как двухсолитонное решение. Исследуем решение уравнения (2), удовлетворяющее граничному условию

$$
\theta(\xi \rightarrow-\infty)=0, \quad \theta(\xi \rightarrow+\infty)=\pi, \quad \theta_{\xi}(|\xi| \rightarrow \infty)=0 .
$$

Для этого считаем $\varphi=\varphi(\tau), \theta=\theta(\xi, \tau)$. Решение системы (2) в случае отсутствия поля $(h=0)$ и затухания $(\alpha=0)$ и удовлетворяющее (3) имеет вид

$$
\begin{aligned}
\operatorname{tg}^{2}(\theta / 2)= & \frac{1-\Omega}{\Omega+\varepsilon \sin ^{2} \varphi} \cdot \frac{1}{\operatorname{ch}^{2}(\sqrt{1-\Omega} \cdot \xi)}, \\
& \left(-\varepsilon \sin ^{2} \varphi<\Omega<1\right)
\end{aligned}
$$

где зависимость угла $\varphi=\varphi(\tau)$ (при $\alpha \ll 1$ и $h \ll 1)$ определяется решением уравнения

$$
\varphi_{\tau}=\Omega+\varepsilon \sin ^{2} \varphi
$$

При условии

$$
\Omega<0,
$$

$\Omega+\varepsilon \sin ^{2} \varphi>0$

решение уравнения (5) для угла $\varphi$ определяется из выражения

$$
\sin ^{2} \varphi=-\frac{\Omega}{\Omega+\varepsilon} \cdot \frac{\operatorname{cth}^{2} \sqrt{-\Omega(\Omega+\varepsilon)} \tau}{1-\Omega /(\Omega+\varepsilon) \operatorname{cth}^{2} \sqrt{-\Omega(\Omega+\varepsilon)} \tau} .
$$

В случае малых полей и затуханий $(h \ll 1, \alpha \ll 1)$, считая в решении (4) параметр $\Omega=\Omega(\tau)$ медленно меняющейся функцией времени, можно получить следующую систему обыкновенных дифференциальных уравнений [11]

$$
\begin{aligned}
& \Omega_{\tau}=\frac{2(1-\Omega)}{\left(1+\alpha^{2}\right)\left(1+\varepsilon \sin ^{2} \varphi\right)} \cdot \\
& \left\{\alpha\left(\Omega+\varepsilon \sin ^{2} \varphi\right)\left(\Omega+\varepsilon \sin ^{2} \varphi-h\right)+\frac{\varepsilon}{2}\left(h+\frac{\alpha \varepsilon}{2} \sin 2 \varphi\right) \sin 2 \varphi\right\} \\
& \cdot\left\{1+\frac{\Omega+\varepsilon \sin ^{2} \varphi}{2 \sqrt{(1-\Omega)\left(1+\varepsilon \sin ^{2} \varphi\right)}} \ln \frac{\left(\sqrt{1-\Omega}+\sqrt{1+\varepsilon \sin ^{2} \varphi}\right)^{2}}{\left|\Omega+\varepsilon \sin ^{2} \varphi\right|}\right\} \\
& \varphi_{\tau}=\frac{1}{1+\alpha^{2}}\left(\Omega+\varepsilon \sin ^{2} \varphi-h-\frac{\alpha \varepsilon}{2} \sin 2 \varphi\right)
\end{aligned}
$$

Система (6) описывает эволюцию параметров $\Omega$ и $\varphi$ солитонного решения (4) под действием поля $h$ и затухания $\alpha$.

Как показывает численный анализ системы (6), в зависимости от начальной амплитуды зародыша возможны два сценария развития: затухание солитона и распад солитона на две 180-градусные доменные стенки (солитон и антисолитон) одинаковой полярности. Подробно это рассмотрено в $[3,11]$.

Образовавшиеся в результате распада зародыша перемагничивания доменные стенки со временем приближаются к устойчивому состоянию, определяемому особой точкой системы (6) 


$$
\sin ^{2} \varphi_{0}=-2 h /(\alpha \varepsilon)=-H / H_{w}, \quad \Omega_{0}=-\varepsilon \sin ^{2} \varphi_{0} .
$$

Эта особая точка существует в полях $|H|<H_{w}$, меньших полей Уокера $H_{w}=2 \pi M_{s} \alpha$, и является устойчивым узлом при $H>0$, а при $H<0$ представляет собой седло. Вблизи этой точки решение (4) принимает вид

$$
\begin{aligned}
\theta= & 2 \operatorname{arctg}\left\{\exp \left\lfloor\left(1+\varepsilon \sin ^{2} \varphi_{0}\right)^{1 / 2}\left(\xi+X_{0}\right)\right]\right\}- \\
& -2 \operatorname{arctg}\left\{\exp \left\lfloor\left(1+\varepsilon \sin ^{2} \varphi_{0}\right)^{1 / 2}\left(\xi-X_{0}\right)\right]\right\}, \\
X_{0}= & \frac{1}{2 \sqrt{1+\varepsilon \sin ^{2} \varphi_{0}}} \ln \frac{1+\varepsilon \sin ^{2} \varphi_{0}}{\left|\Omega+\varepsilon \sin ^{2} \varphi_{0}\right|},
\end{aligned}
$$

где $\Omega+\varepsilon \sin ^{2} \varphi_{0} \rightarrow 0$.

Это решение описывает взаимодействующие 180 -градусные стенки одинаковой полярности, расстояние между которыми $\left(L=2 X_{0} \rightarrow \infty\right)$ резко возрастает при $H>0$ и $\varphi \rightarrow \varphi_{0}, \Omega \rightarrow-\varepsilon \sin ^{2} \varphi_{0}$. В случае противоположного направления поля, т.е. $H<0$, с ростом $\Omega+\varepsilon \sin ^{2} \varphi$ две 180 -градусные стенки одинаковой полярности сближаются и должно произойти рассеяние или аннигиляция доменных стенок в зависимости от соотношения величин поля $h$ и параметра затухания $\alpha$.

Для анализа этого процесса систему (6) перепишем в новых переменных $V$ и Ф согласно выражению

$$
\begin{aligned}
& \Omega=-\frac{2 V^{2} / \varepsilon}{1-V^{2} / \varepsilon+\sqrt{\left(1-V^{2} / \varepsilon\right)^{2}-4 V^{2} / \varepsilon^{2}}}, \\
& \sin ^{2} \varphi=-\frac{\Omega}{\Omega+\varepsilon} \cdot \frac{\operatorname{cth}^{2} \Phi}{1-\Omega /(\Omega+\varepsilon) \operatorname{cth}^{2} \Phi} .
\end{aligned}
$$

Здесь $V$ - скорость солитона и антисолитона относительно их общего центра; $\Phi$ - характеризует расстояние между ними. Эти параметры являются медленно меняющимися функциями времени и определяются из уравнений

$$
\begin{aligned}
\Phi_{\tau}= & -\frac{1}{V\left(1+\alpha^{2}\right)}\left[\sqrt{1-\Omega} J_{3}+\frac{2 \operatorname{sh} \Phi \operatorname{ch} \Phi J_{2}}{1-V^{2} / \varepsilon+J_{1}}\right], \\
V_{\tau}= & -\frac{2 J_{1} J_{2}}{\left(1+\alpha^{2}\right)\left(1-V^{2} / \varepsilon+J_{1}\right) J_{4}}, \\
J_{1}= & \sqrt{\left(1-V^{2} / \varepsilon\right)^{2}-4 V^{2} / \varepsilon^{2}} \\
J_{2}= & \left(\alpha V\left(h+\frac{V^{2}(1-\Omega)}{\Omega-\varepsilon \operatorname{sh}^{2} \Phi}\right)+\right. \\
& \left.+\frac{\varepsilon}{\sqrt{1-\Omega}}\left(h+\alpha \varepsilon \frac{V \sqrt{1-\Omega}}{\Omega-\varepsilon \operatorname{sh}^{2} \Phi} \operatorname{sh} \Phi \operatorname{ch} \Phi\right) \operatorname{sh} \Phi \operatorname{ch} \Phi\right) \times \\
& \quad\left(1-\frac{\Omega}{2 \sqrt{(1-\Omega) J_{4} J_{5}}} \ln \frac{\left(\sqrt{(1-\Omega) J_{5}}+\sqrt{J_{4}}\right)^{2}}{\Omega}\right)
\end{aligned}
$$

$J_{3}=V^{2}+h \frac{\Omega-\varepsilon \operatorname{sh}^{2} \Phi}{1-\Omega}+\alpha \varepsilon \frac{V}{\sqrt{1-\Omega}} \operatorname{sh} \Phi \operatorname{ch} \Phi$

$J_{4}=\operatorname{sh}^{2} \Phi-(1+\varepsilon) \frac{\Omega}{\Omega+\varepsilon} \operatorname{ch}^{2} \Phi$,

$J_{5}=\operatorname{sh}^{2} \Phi-\frac{\Omega}{\Omega+\varepsilon} \operatorname{ch}^{2} \Phi$.

Из этой системы можно получить следующее уравнение, определяющее зависимость скорости $V$ от $\Phi$, т.е. $V=V(\Phi)$ :

$$
\frac{d V}{d \Phi}=\frac{2 V J_{1} J_{2}}{\sqrt{1-\Omega}\left(1-V^{2} / \varepsilon+J_{1}\right) J_{3} J_{4}+2 \operatorname{sh} \Phi \operatorname{ch} \Phi J_{2}}
$$

Численное исследование системы (10) с начальными условиями

$$
\Phi_{0} \rightarrow-\infty, \quad V_{0}=\frac{h / \alpha}{\sqrt{1+\varepsilon\left(1+\sqrt{1-4 h^{2} / \alpha^{2} \varepsilon^{2}}\right) / 2}}
$$

показывает сильную зависимость процесса взаимодействия стенок в магнитном поле от затухания. На рис. 1 показана фазовая диаграмма процесса взаимодействия стенок в поле $h<0$. Из рисунка видно, что стенки движутся из бесконечности друг на друга с постоянной скоростью, когда они сближаются, происходит взаимодействие, после чего продолжают двигаться с том же направлении только с уменьшающейся со временем скоростью. Такое замедление их движения происходит за счет силы со стороны магнитного поля. После чего стенки остановятся на некотором расстоянии и впоследствии начинают двигаться друг навстречу другу. Таким образом, солитон-антисолитонная пара (пара 180-градусных стенок одинаковой полярности) прежде чем аннигилировать, испытывают многократный процесс рассеяния, сопровождающийся сменой знака Ф, то есть полярности 180-градусных стенок. После каждого последующего акта максимальное расстояние, на которое разойдутся стенки, то есть ширина домена обратной намагниченности, уменьшается. Аналогичная ситуация имеет место и в слабых ферромагнетиках [12]. Эволюция формы взаимодействующих доменных стенок одинаковой полярности при $h<0$ приведена на рис. 2. Следует заметить, что описанный выше процесс происходит ниже критического значения внешнего магнитного поля при заданном параметре затухания.

Расстояние между стенками можно определить как расстояние между точками перегиба кривой $\theta=\theta(\xi)$, задаваемой (4). Тогда получим выражение:

$$
T=\frac{2}{\sqrt{1-\Omega}} \operatorname{arcsh} \sqrt{\frac{1+\varepsilon \sin ^{2} \varphi}{\Omega+\varepsilon \sin ^{2} \varphi}} .
$$

Зависимость от времени расстояния $T=T(\tau)$ между стенками относительно их общего неподвижного центра при разном затухании приведена на рис. 3. Временной интервал на этом рисунке ограничен двукратным взаимодействием стенок, как и на рис. 2. Численные расчеты показывает, что расстояние между стенками $T$ зависит от величины внешнего магнитного поля и параметра затухания. Максимальное расстояние между стенками, на которое они разойдутся после взаимодействия, будет уменьшаться с уменьшением значения внешнего поля и увеличением параметра затухания.

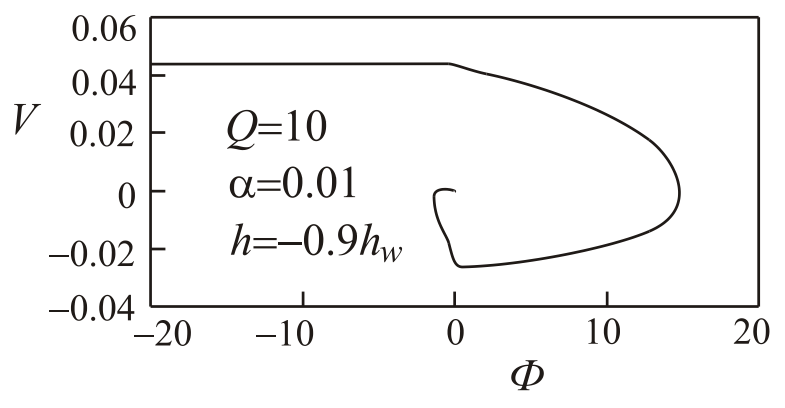

Рис. 1. Фазовая диаграмма взаимодействия стенок.

Fig. 1. Phase diagram of the wall interaction. 


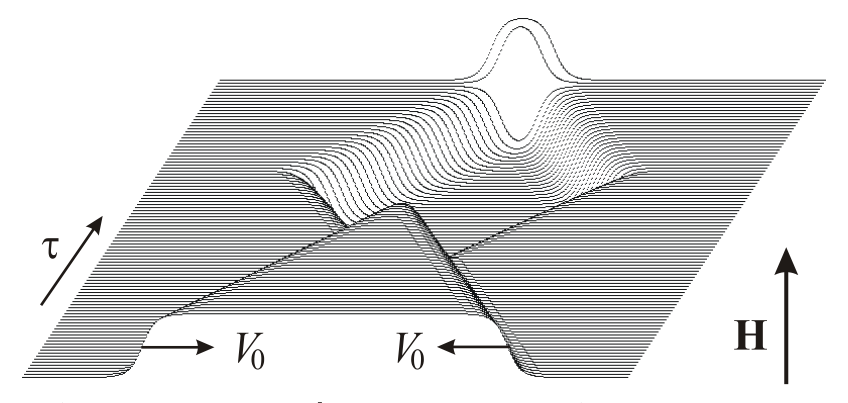

m个 $\mathbf{m} \downarrow$ m 个

Рис. 2. Взаимодействие 180-градусных доменных стенок, сопровождающееся их аннигиляцией, при $h=-0.9 h_{w}, Q=10$, $V_{0}=0.04$ и $\alpha=0.01$.

Fig. 2. Interaction of 180-degree domain walls accompanied by their annihilation, at $h=-0.9 h_{w}, Q=10$, initial velocity $V_{0}=0.04$ and damping parameter $\alpha=0.01$.

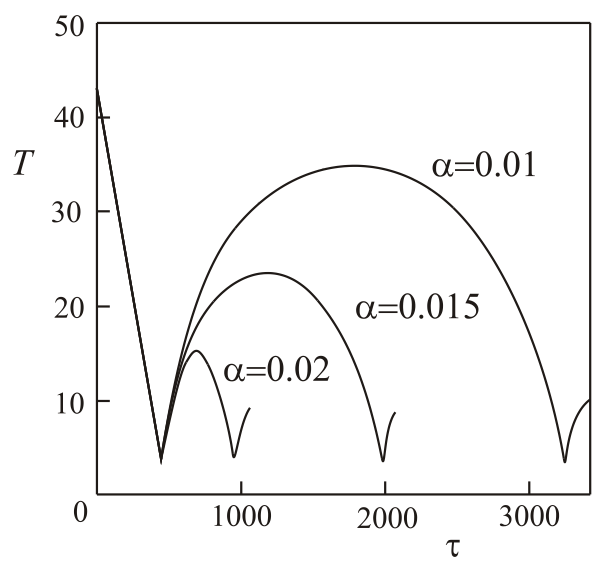

Рис. 3. Зависимость расстояния между стенками $T$ от времени для разных значений параметра затухания $\alpha$ при $h=-0.9 h_{w}$, $Q=10$, начальной скорости $V_{0}=0.04$.

Fig. 3. Dependence of the distance between the walls $T$ on time with a different damping parameter $\alpha$ at $h=-0.9 h_{w}, Q=10$, initial velocity $V_{0}=0.04$.

Известно, что взаимодействующие кинк и антикинк не претерпевают каких либо изменений, то есть после взаимодействия имеют такую же форму, амплитуду и скорость. Единственный результат взаимодействия это фазовый сдвиг. Так как наши доменные стенки описываются решением кинка и антикинка, то результатом взаимодействия стенок будет только фазовый сдвиг. То есть при взаимодействии изменяется координата центра стенки $X_{0}$. На рис. 4 представлена временная зависимость координаты центра одной стенки, где через $\Delta x$ обозначен фазовый сдвиг.

Полученные результаты численного счета показывают, что фазовый сдвиг $\Delta x$ не зависит от параметра $\alpha$, практически не зависит от величины $h$, но имеет хорошую зависимость от фактора качества материала $Q$. А именно, с ростом $Q$ увеличивается и величина $\Delta x$ (рис. 5 ).

С увеличением параметра $\alpha$, а именно, выше определенного его значения, в поле $h<0$ аннигиляция солитон-антисолитонной пары (пары 180-градусных стенок одинаковой полярности) произойдет в результате одноактного процесса рассеяния (рис. 6). Процесс аннигиля- ции доменных стенок, как для случая одноактного, так и для многоактного процесса, можно наблюдать для линейно двойных систем [13].

В случае поля противоположного направления поля $0<H<H_{\text {w }}$ на движущиеся навстречу друг другу стенки кроме тормозящей силы действует еще отталкивающая сила со стороны поля. При этом доменные стенки приближаются на минимальное расстояние, после чего начинается обратный процесс их разбегания друг от друга (рис. 7).

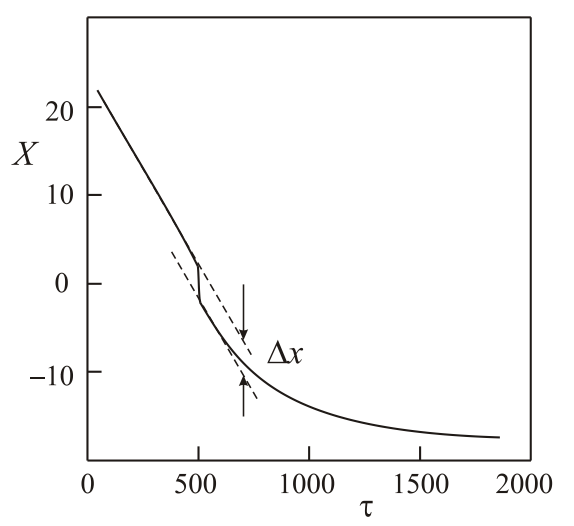

Рис. 4. Зависимость значения координаты центра стенки $X$ от времени и фазовый сдвиг $\Delta x$ при взаимодействии.

Fig. 4. Dependence of the wall centre coordinate $X$ on time and the phase shift $\Delta x$ during interaction.

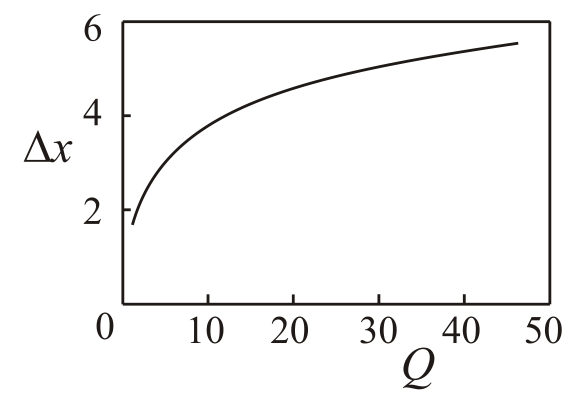

Рис. 5. Зависимость фазового сдвига $\Delta x$ от фактора качества материала $Q$.

Fig. 5. Dependence of phase shift on the material quality factor.

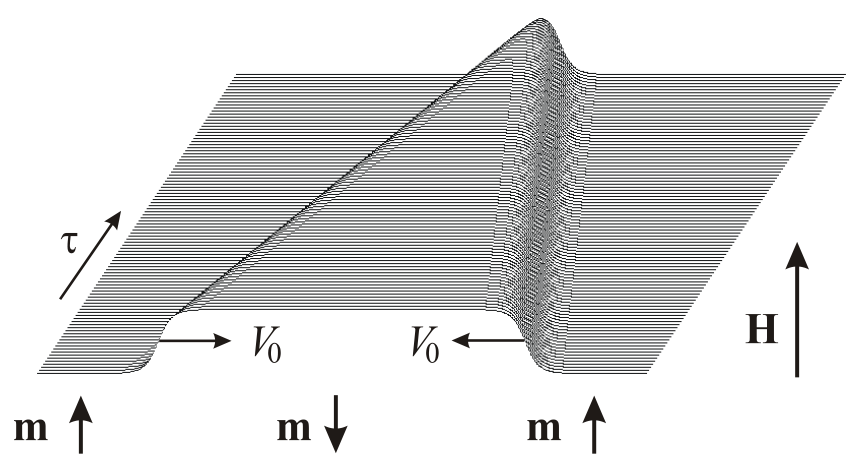

Рис. 6. Взаимодействие 180-градусных доменных стенок, сопровождающееся их аннигиляцией, в результате одноактного процесса рассеяния при $h=-0.9 h_{w}, Q=10, V_{0}=0.04$ и $\alpha=0.2$.

Fig. 6. Interaction of 180-degree domain walls accompanied by their annihilation as a result of the single-act dissipation at $h=-0.9 h_{w}$, $Q=10$, initial velocity $V_{0}=0.04$ and damping parameter $\alpha=0.2$. 


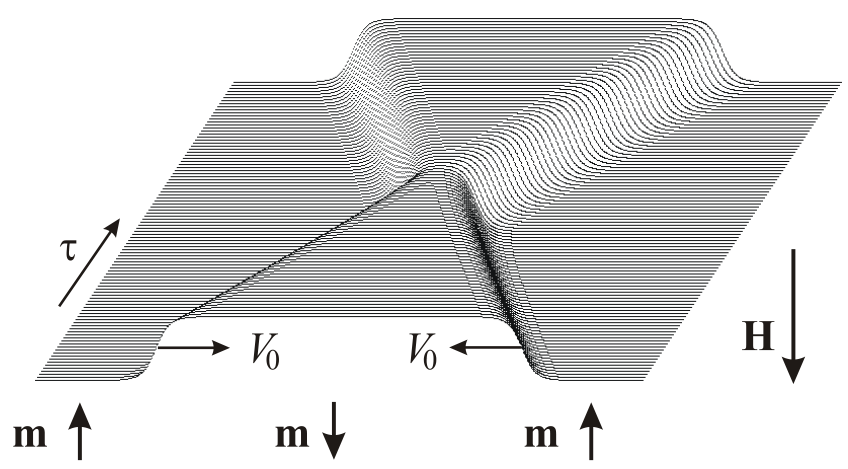

Рис. 7. Взаимодействие 180-градусных доменных стенок в поле $h>0$ с параметрами и начальными условиями, что и на рис. 2.

Fig. 7. Interaction of 180-degree domain walls in the field $h>0$ with the parameters and initial conditions same as those in Fig. 2.

В заключении отметим, что при взаимодействии двух 180-градусных стенок одинаковой полярности в зависимости от направления внешнего магнитного поля может иметь место аннигиляция стенок в результате либо одноактного, либо многократного процесса рассеяния, или сближение, отталкивание и последующее разбегание стенок.

Авторы посвящают статью семидесятилетию учителя - проф. Шамсутдинова М. А., являвшегося инициатором и вдохновителем данного направления исследований.

\section{Литература/References}

1. M. A. Shamsutdinov, I. Yu. Lomakina, V.N. Nazarov, et al. Ferro- and Antiferromagnetodynamics. Nonlinear Oscillations, Waves, and Solitons. Moscow, Nauka. (2009) 456 p. (in Russian) [M.А. Шамсутдинов, И. Ю. Ломакина, В.Н. Назаров и др. Ферро- и антиферромагнитодинамика. Нелинейные колебания, волны и солитоны. Москва, Наука. 2009. 456 с].

2. Ekomasov E.G., Gumerov A.M., Murtazin R.R., KudryavtsevR. V., Ekomasov A. E., Abakumova N. N.Solid State Phenomena. Switzerland. Trans Tech Publications. 233 - 234, 51 - 54 (2015).

3. Ekomasov E.G., Gumerov A.M. Letters on Materials. 4 (4) 237-240 (2014). (in Russian) [Е.Г. Екомасов, А. М. Гумеров. Письма о материалах. 4 (4), $237-240$ (2014).]

4. Sug-Bong Choe, Sung-Chul Shin. Journal of Magnetism and Magnetic Materials. 236, 249 - 254 (2001).

5. S. Wiebel, J.-P. Jamet, N. Vernier, A. Mougin and J. Ferré. Journal of Applied Physics. 100, 043912 (2006).

6. A. Berger, Physica B. 407, $1322-1329$ (2012).

7. G. S. Kandaurova. Physics-Uspekhi. 45, 1051 (2002). (in Russian) [Г.С. Кандаурова. Успехи физических наук. 172, 1165 (2002)].

8. Şt. C. Bădescu, V. Bădescu, N. Rezlescu, Rodica Bădescu. Sensors and Actuators A: Physical. 81, 244-247 (2000).

9. W. M. Liu, B. Wu, X. Zhou, D. K. Campbell, S. T. Chui and Q. Niu. Phys. Rev. B. 65, 172416 (2002).

10. A. M. Kosevich. Low Temp. Phys. 27, 513 (2001). (in Russian) [А. М. Косевич. Физика низких температур. 27, 699- 737 (2001)].

11. M. A. Shamsutdinov, V. N. Nazarov, and I. Yu. Lomakina. Phys. Met. Metallogr. 101, 309 (2006). (in Russian) [М. А. Шамсутдинов, В.Н. Назаров, И. Ю. Ломакина. Физика металлов и металловедение. 101, 339-349 (2006)].

12. M. A. Shamsutdinov, I. Yu. Lomakina, and V. N. Nazarov. Phys. Met. Metallogr. 100, 541 (2005). (in Russian) [М. А. Шамсутдинов, И. Ю. Ломакина, В. Н. Назаров. Физика металлов и металловедение. 100, 17-33 (2005).]

13. Nir Dror, Boris A. Malomed, and Jianhua Zeng. Phys. Rev. E. 84, 046602 (2011). 